\title{
BMJ Open Assessing cardiovascular remodelling in fetuses and infants conceived by assisted reproductive technologies: a prospective observational cohort study protocol
}

Wen-jing Bi, Li Cui, Yang-jie Xiao, Guang Song, Xin Wang, Lu Sun, Wei Qiao, Wei-dong Ren (1D)

To cite: Bi W, Cui L, Xiao Y, et al. Assessing cardiovascular remodelling in fetuses and infants conceived by assisted reproductive technologies: a prospective observational cohort study protocol. BMJ Open 2019;9:e031452. doi:10.1136/ bmjopen-2019-031452

- Prepublication history for this paper is available online. To view these files, please visit the journal online (http://dx.doi. org/10.1136/bmjopen-2019031452).

Received 06 May 2019 Revised 06 0ctober 2019 Accepted 09 October 2019
Check for updates

(C) Author(s) (or their employer(s)) 2019. Re-use permitted under CC BY-NC. No commercial re-use. See rights and permissions. Published by BMJ.

Department of Ultrasound, Shengjing Hospital of China Medical University, Shenyang, China

Correspondence to Professor Wei-dong Ren; renwdcmu@163.com

\section{ABSTRACT}

Introduction Assisted reproductive technologies (ART), namely in vitro fertilisation and intracytoplasmic sperm injection, have become widely used to treat infertility. Although the use of ART is generally considered favourable, there are ongoing concerns about the prenatal and perinatal risks as well as long-term risks for the child. Epidemiological studies have demonstrated an association between pathological events during fetal development and future cardiovascular risk, raising concerns about cardiovascular remodelling in fetuses conceived by ART. The authors hypothesise fetuses conceived by ART present signs of cardioventricular dysfunction, which can be detected by deformation analysis. To address these issues, we will assess comprehensive cardiovascular structure and function in ART offspring and explore the role of speckle-tracking in myocardial deformation.

Methods and analysis This prospective observational cohort study will include 100 singleton pregnancies conceived by ART and 100 controls identified in fetal life and followed up to 6 months old. At inclusion, a baseline assessment of the mothers and ART characteristics will be recorded by interview and review of medical records. Between 28 and 32 weeks gestation, a detailed fetal echography will be performed, including an assessment of estimated fetal weight, fetoplacental Doppler, fetal echocardiography and fetal abdominal artery ultrasound. On delivery, maternal and neonatal characteristics will be assessed. Within 60 days of birth, the first postnatal cardiovascular assessment will be conducted which will include echocardiography and abdominal artery ultrasound. At 6 months of age, the second infants' follow-up evaluation will include the weight and length of the infant, echocardiography and abdominal artery ultrasound. Data will be presented as mean $\pm \mathrm{SD}$, median or percentages where appropriate. $A p<0.05$ will be considered statistically significant.

Ethics and dissemination Ethical approval has been obtained from the Ethics Committee of Shengjing Hospital of China Medical University. Findings will be disseminated through scientific publications and conference presentations.

Trial registration number ChiCTR1900021672.
Strengths and limitations of this study

- This is one of the first studies to assess cardiovascular remodelling in fetuses and infants conceived by assisted reproductive technologies.

- There are few studies that focus on biventricular systolic and diastolic function of fetuses using a comprehensive cardiac functional assessment and exploring the role of speckle-tracking to assess myocardial deformation.

- There are few studies that focus on fetal ventricular twist and untwist process by strain tracking echocardiography.

- As a single-centre, non-randomised, prospective observational cohort study, the universality of the result may be limited. It should be replicated at other centres and in other patient populations.

- Potential loss to follow-up may lead to some loss of data.

\section{INTRODUCTION}

Assisted reproductive technologies (ART), which include in vitro fertilisation (IVF) and intracytoplasmic sperm injection (ICSI), are now widely allowed for pregnancy in cases of infertile couples. Since the first child (Louise Brown) was born after IVF 40 years ago, the number of assisted pregnancies has increased exponentially. ${ }^{1}$ At present, $1 \%-6 \%$ of children born in all regions of the world are conceived by ART, with an increasing application of about 1 million babies born using ART annually. ${ }^{23}$ Although the use of ART is generally considered safe, increasing research focuses on the prenatal and perinatal risks as well as long-term risks for the child. ${ }^{45}$

A growing body of evidence demonstrates the potential for adverse perinatal outcomes in ART pregnancies, including preterm birth, low birth weight, congenital malformations 
and perinatal mortality. ${ }^{6-8}$ Although parental predisposing infertility factors and other maternal factors may contribute to these adverse outcomes, ${ }^{9}{ }^{10}$ the ART procedure itself may play a role. This includes the exposure of oocytes, sperm and embryos outside of the body environment, freezing and manipulation of oocytes and embryos. ${ }^{4511}$ Animal studies have highlighted the biological plausibility of imprinted disorders in the placenta and cord blood of offspring conceived by ART. ${ }^{12}$

However, the absolute risks of adverse perinatal outcomes are low. We should pay more attention to the apparently healthy offspring conceived by ART. Among the potential long-term consequences of ART, concern has been raised regarding cardiovascular disease. Epidemiological studies in humans have demonstrated an association between adverse events during early life and increased prevalence of cardiovascular disease later in life. The term 'fetal programming of cardiovascular disease' was coined to describe this hypothesis. ${ }^{13} 14$ Growing evidence has suggested that ART represents another example for fetal cardiovascular remodelling. Ceelen et al first suggested the presence of elevated blood pressure during late childhood among individuals conceived by ART. ${ }^{15}$ Recent study also demonstrated decreased vascular function in adolescence conceived by ART. ${ }^{16}$ Furthermore, studies of children conceived by ART indicated adverse changes in systemic and pulmonary circulations, including both structural and functional alterations, some of which have been prospectively linked to arterial hypertension and premature cardiovascular morbidity. ${ }^{17}$ Further research found a possible association between ART and cardiovascular remodelling in fetal life that persisted in postnatal life. ${ }^{418}$ In mice generated by ART, alteration of the cardiovascular phenotype was mediated by an epigenetic mechanism which alters the methylation of the promoter of the gene coding for endothelial nitric oxide synthase. This leads to endothelial dysfunction and premature vascular senescence. ${ }^{19}$ Moreover, ART also had consequences on the life span of mice. When challenged with a high-fat diet, the life span of ART mice was shortened by approximately $25 \%$ compared with control mice. ${ }^{19}$ While these studies are very informative, the commonly applied evaluation methods provide only indirect information about systolic and diastolic ventricular function, and do not measure myocardial deformation itself.

There are very few studies to date that have focused on cardiac myocardial deformation using echocardiography in offspring conceived by ART. Valenzuela-Alcaraz et al first suggested cardiac dysfunction and remodelling in ART fetuses, and these problems persisted postnatally. ${ }^{4}$ This study showed dilated atria, increased myocardial wall thickness, lower ventricular sphericity indexes, decreased right longitudinal function and impaired relaxation (decrease in $\mathrm{E}^{\prime}$ and $\mathrm{E}$ deceleration time) in ART fetuses. ${ }^{4}$ These changes persisted postnatally and were more prominent in the right side of the heart. ${ }^{4}$ However, ventricular function is increasingly understood not simply as myocyte shortening, but also as a more complex process, including longitudinal contraction, circumferential contraction, radial contraction and twist-untwist process. The commonly applied evaluation methods provide only global information about systolic and diastolic function and do not measure deformation of the myocardium itself. More recently, speckle-tracking echocardiography (STE) has been developed to overcome these limitations. This technique directly analyses myocardial mechanics by tracking the tissue pixels in the myocardium throughout the cardiac cycle. This technique has the potential to provide new insights into subclinical changes in fetal myocardial deformation. ${ }^{20} 21$

Our objectives are to assess the effect of ART on offspring's cardiovascular health in fetal and postnatal periods, namely (1) using commonly applied evaluation methods and (2) exploring the potential role of STE on cardiac functional assessment. The aim of this study is to further understand the potential adverse effects of ART, which will allow clinicians to work towards improving ART-related cardiovascular outcomes.

\section{METHODS AND ANALYSIS Study design}

This will be a prospective observational cohort study including 100 singleton pregnancies conceived by ART and 100 spontaneously conceived controls identified in fetal life and followed up to 6 months old. Cases and controls were recruited from December 2017 to April 2019 at the Department of Ultrasound, Shengjing Hospital of China Medical University, Shenyang, China. The expected deadline of last follow-up is November 2019. Conception by ART includes standard IVF and ICSI.

\section{Patient and public involvement}

Patients and the public will not be involved in this study.

\section{Inclusion and exclusion criteria}

The inclusion criteria for the ART group are singleton pregnancies of mothers aged 18 years or older, conceived by ART, and are at 28-32 weeks of gestation. The exclusion criteria for ART group are presence of preimplantation genetic diagnosis, oocyte donation, pregnancies with structural/chromosomal anomalies, evidence of infection or any maternal medical disease including asthma, chronic hypertension, diabetes mellitus, heart disease, HIV or hepatitis infection, lupus and thyroid disease. Likewise, delivery before 37 weeks of gestation and birth weight below the 10th or above the 90th percentile are considered additional exclusion criteria, in order to avoid the underlying interference of prematurity and small/ large-for-gestational age. Small/large-for-gestational age is defined as birth weight below the 10th or above the 90th centile according to local reference curves. ${ }^{22}$ Moreover, smoking during pregnancy will be additional excluding criteria. The control group will also be recruited at 28-32 weeks of gestation and matched for maternal age $( \pm 1$ year) with ART cases. Inclusion and exclusion criteria 
for control group are the same as for ART group, and the control group will undergo the same study protocol as ART group. The gestational age of both groups is calculated according to crown-rump length at first-trimester scan.

\section{Sample size calculation}

As a primary outcome, the global longitudinal strain was used to calculate sample size using Power and Sample Size Calculation Software. ${ }^{23}$ Assuming that left ventricle (LV) global longitudinal systolic strain (\%) will be -16.99 (SD: 2.42) in fetuses from the control group ${ }^{20}$ compared with -15.78 (SD: 2.42) in those of ART group, the required sample size is 65 fetuses in both groups to have a statistically significant difference $(\mathrm{p}<0.05$, two-sided $\mathrm{t}$-test $)$ with a power of $80 \%$. Considering the effect of dropouts and other confounding factors, we plan to include 100 fetuses in both groups.

\section{Recruitment procedure}

During pregnancy, data for all pregnant women who meet the inclusion criteria will be obtained from Shengjing Hospital of China Medical University Information Management System. These women will be telephoned by a trained data collector and briefly introduced the purpose of this study. For those volunteers who give consent to the follow-up call, a two-dimensional fetal echocardiographic study and fetal vascular imaging will be scheduled.

At the time of the prenatal evaluation, parental baseline and ART characteristics will be recorded by interview and a review of medical records, including parental ethnicity, age, body mass index, smoking habit, nulliparity, socioeconomic status, college education and ART characteristics. Family cardiovascular history is defined as the existence of congenital heart disease, coronary disease, hypertension, diabetes, hypercholesterolemia or stroke in men $<55$ years and women $<65$ years. Written informed consent will be obtained from all study participants. All pregnant women will undergo a detailed fetal echocardiography by the same experienced operator $(\mathrm{W}-\mathrm{j} B)$ to exclude congenital heart disease. A standard assessment of estimated fetal weight, fetoplacental Doppler, comprehensive fetal echocardiography and fetal abdominal artery ultrasound will be added to routine fetal echography.

On delivery, presence of pregnancy complications, gestational age at delivery, mode of delivery, maternal blood pressure, neonatal sex, birth weight, birth weight centile, birth length and perinatal morbidity will be recorded. Major neonatal morbidity is defined as the existence of bronchopulmonary dysplasia, necrotising enterocolitis, intraventricular haemorrhage, periventricular leukomalacia, retinopathy, persistent ductus arteriosus or sepsis in the first 28 days of life. Within 60 days of birth, the first postnatal cardiovascular assessment will be conducted, which will include echocardiography and an abdominal artery ultrasound. At 6 months of age, infants' follow-up evaluation will include the weight and length of the infant, echocardiography and an abdominal artery ultrasound assessment. The operators obtaining the echographic images and analysing image data will be blinded to the group assignment to avoid information bias.

\section{Fetal assessment}

All pregnancies will undergo ultrasonic examination at 28-32 weeks of gestation using an EPIQ 7 ultrasound systems (Philips Medical Systems) equipped with 3-5 MHz curved-array (C5-1), 1-5 MHz sector-array (S5-1) and $3-10 \mathrm{MHz}$ linear-array probes $(9 \mathrm{~L})$. Ultrasounds will include an assessment of estimated fetal weight, fetoplacental Doppler, fetal echocardiography and fetal abdominal artery ultrasound. Fetal M-mode, B-mode, and Pulsed-wave (PW) Doppler measurements will be obtained with the curved-array probe, while the sectorarray probe will be used for the tissue Doppler examination. The fetal abdominal artery ultrasound assessment will be recorded with the linear-array probe. PW Doppler parameters will be recorded from three or more waveforms, with insonation angle $<60^{\circ}$ for the fetoplacental Doppler evaluation and $<30^{\circ}$ for the fetal echocardiography assessment.

\section{Fetoplacental Doppler evaluation}

The fetoplacental Doppler evaluation will include pulsatility index (PI) measurement of the uterine arteries, umbilical artery, middle cerebral artery, ductus venosus and aortic isthmus according to a former published methodology. ${ }^{24-27}$ The cerebroplacental ratio will be obtained by dividing middle cerebral artery and umbilical artery $\mathrm{PI}^{28}$

\section{Fetal echocardiography}

Fetal echocardiography will include not only a conventional morphometric and functional assessment but also a deformation analysis.

\section{Fetal cardiac morphometry}

Fetal cardiac morphometry will include cardiothoracic ratio, atrial and ventricular areas, ventricular sphericity indices of base, and mid-section and relative wall thicknesses. The cardiothoracic ratio will be obtained from a 4-chamber view and calculated from the ratio of heart area to thoracic area. ${ }^{29}$ Atrial and ventricular areas will be measured at the maximum point of atrial or ventricular distension. Biventricular sphericity indices of the base section will be calculated as the ratios of base-toapex length/basal ventricular diameters and measured at end-diastole. ${ }^{30} 31$ Biventricular sphericity indices of the mid-section will be calculated as the ratios of base-toapex length/transverse length at the mid-section of the ventricle and measured at end-diastole. ${ }^{32}$ The relative wall thickness will be calculated for the left and right ventricular walls, with the formula ( $2 \times$ wall thickness)/(ventricular inner diameter at end-diastole).$^{33}$ 


\section{Fetal cardiac function}

The conventional fetal cardiac function evaluation will include biventricular systolic and diastolic function, and biventricular global function. Assessment of systolic function will include left/right ejection fraction, stroke volumes, cardiac outputs, mitral/tricuspid annular-plane systolic excursion (MAPSE/TAPSE) and systolic annular peak velocity $\left(S^{\prime}\right)$. Left/right ejection fraction will be calculated from M-mode long-axis transverse 4-chamber view using the Teicholz formula. ${ }^{29}$ Left and right stroke volumes will be obtained as follows: $\Phi / 4 \times$ (aortic or pulmonary valve diameter $)^{2} \times($ aortic or pulmonary artery systolic time-velocity integral). ${ }^{34}$ Left and right cardiac outputs will be obtained as: left or right stroke volume $\times$ heart rate. ${ }^{34}$ MAPSE/TAPSE will be assessed by M-mode from an apical or basal 4-chamber view. ${ }^{35}$ The tissue Doppler will be used to record systolic peak velocities $\left(\mathrm{S}^{\prime}\right)$ at mitral and tricuspid lateral annuli from an apical or basal 4-chamber view. ${ }^{36}$ Assessment of diastolic function will include peak early and late transvalvular filling (E/A) ratio, deceleration time of $\mathrm{E}$ velocity, early (E') diastolic annular peak velocities and left isovolumic relaxation time (IVRT). Atrioventricular flows will be obtained from an apical or basal 4-chamber view, placing the pulsed Doppler sample volume at the tip of atrioventricular valve leaflets. Left and right $\mathrm{E} / \mathrm{A}$ ratios will be calculated by dividing early ventricular filling ( $\mathrm{E}$ wave) by late ventricular filling (A wave). ${ }^{37}$ The deceleration time of the $\mathrm{E}$ wave will be obtained from mitral and tricuspid inflow velocities from a basal or apical chamber view. The tissue Doppler will be used to record early diastolic $\left(E^{\prime}\right)$ peak velocity at mitral and tricuspid lateral annuli from an apical or basal 4-chamber view. Left IVRT will be measured from the closure of the aortic valve to the opening of the mitral valve in an apical or basal long-axis view. The left/right ventricular myocardial performance index (Tei index) will be used to assess the combination of systolic and diastolic function of the LV and the right ventricle $(\mathrm{RV})$, respectively. These will be calculated using pulse Doppler methods ${ }^{38}$ as: $(\mathrm{a}-\mathrm{b}) / \mathrm{b}$, where ' $\mathrm{a}$ ' is the time from the closure click to the aperture click of the mitral/ tricuspid valve, and ' $b$ ' is the time from the aperture click to the closure click of the aortic/pulmonary valve.

\section{STE}

As the position of the fetus is changeable, fetal LV long/ short-axis views are different from the standard postnatal transthoracic parasternal views. Therefore, fetal posture will be unified first. By moving the position of the pregnant women, the head side of all fetuses will be oriented to the end of the examination bed, that is, the lying direction of the pregnant women with breech position will be opposite to that of the pregnant women with cephalic position. According to a strict protocol for every participant, high-resolution, zoomed B-mode digital loops with $7 \mathrm{~s}$ cine clips will be acquired from a slightly angled apical/basal 4-chamber, long-axis and 2-chamber views and basal-, mid- and apical-short-axis views, with frame rate set at more than 80 frames/s. Image-acquisition adjustments, such as narrow sector width, depth reduction, focus or frequency adjustment, will be used to optimise the frame rate. In addition, the lateral walls and interventricular septum will be kept between $0^{\circ}$ and $45^{\circ}$ to the ultrasonic beam in apical/basal long-axis views to enhance the borders between the blood pool and endocardium. Meanwhile, recordings will be obtained during maternal breath holding and in the absence of fetal movements. Based on apical/basal 4-chamber view, the fetal long-axis and 2-chamber view could be obtained by rotating the probe (figure 1). For example, based on apical 4-chamber view, the long-axis view will be obtained by rotating the probe clockwise $60^{\circ}$, and the 2-chamber view will be obtained by continuing the rotation clockwise $30^{\circ}$. Next, based on basal 4-chamber view, the long-axis view will be obtained by rotating the probe clockwise $120^{\circ}$, and the 2-chamber view will be obtained

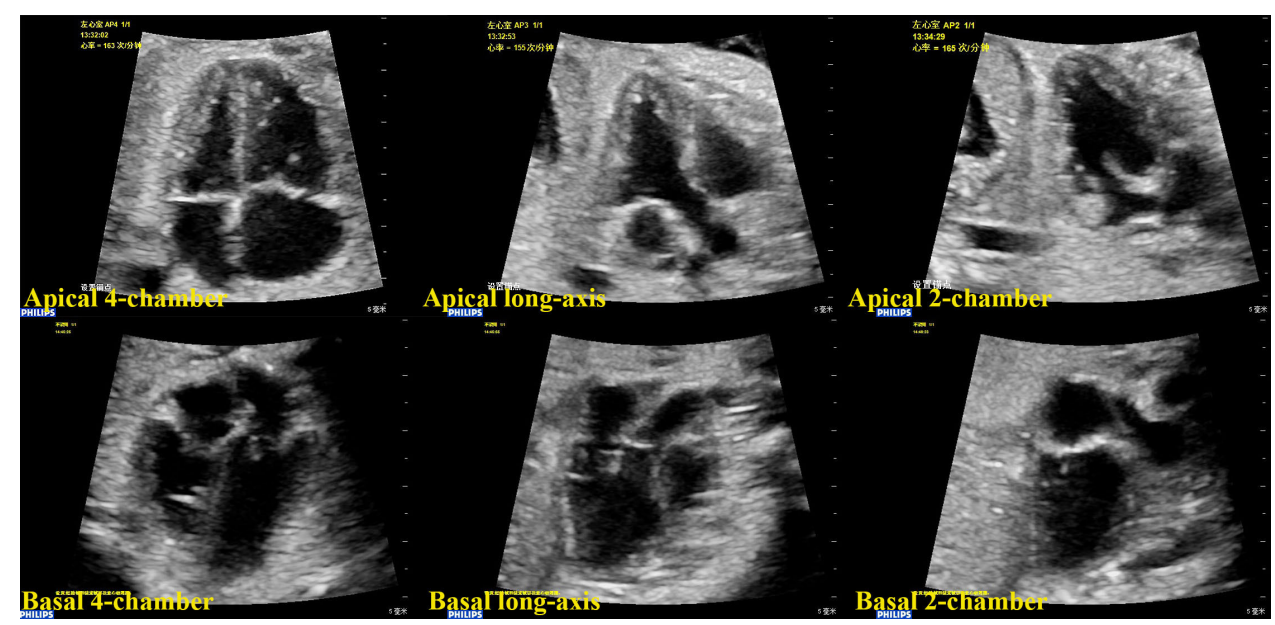

Figure 1 On the basis of apical 4-chamber view, the long-axis view will be obtained by rotating the probe clockwise $60^{\circ}$, and the 2 -chamber view will be obtained by continuing the rotating clockwise $30^{\circ}$. Next, on the basis of basal 4-chamber view, the long-axis view will be obtained by rotating the probe clockwise $120^{\circ}$, and the 2 -chamber view will be obtained by continuing the rotating counterclockwise $30^{\circ}$. 


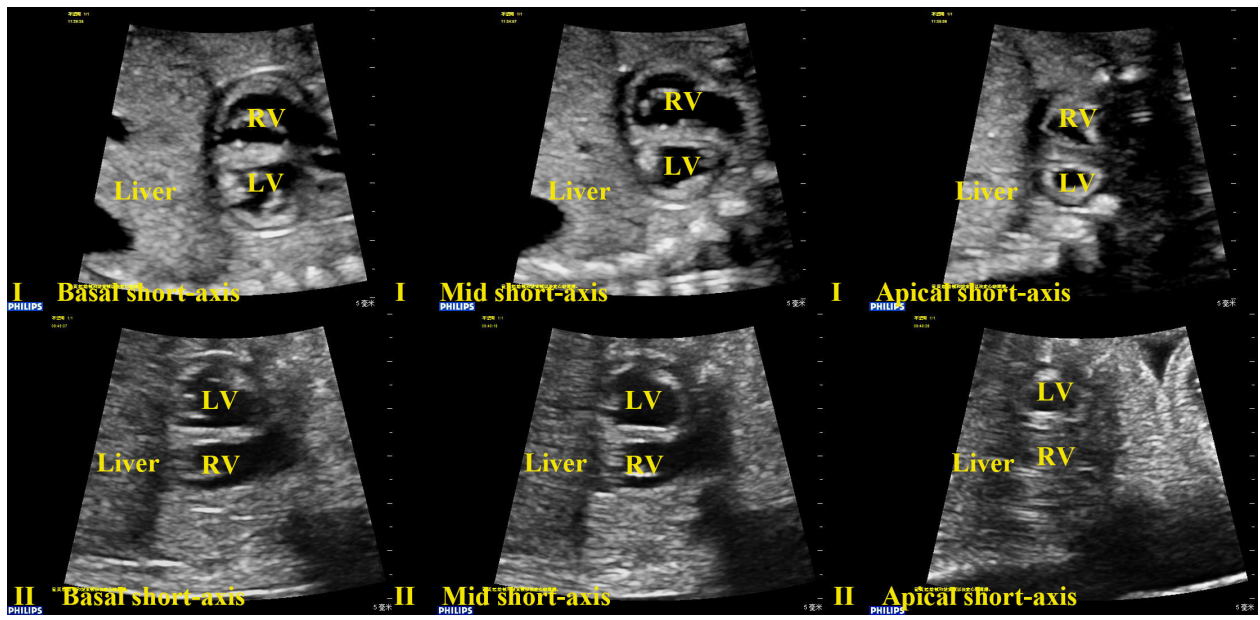

Figure 2 Two types of orientation for fetal heart short-axis images: (I) the liver is toward the 'left of the image' and the left ventricle (LV) is at the 'bottom of the image', similar to the traditional transthoracic short-axis image; (II) the liver is toward the 'left of the image' and the LV is at the 'top of the image'. LV, left ventricle; RV, right ventricle.

by continuing the rotation counterclockwise $30^{\circ}$. The 4-chamber view will then be imaged when the walls and interventricular septum are $90^{\circ}$ to the ultrasound beam and rotating the probe $90^{\circ}$ clockwise. After adjusting the probe slightly, the short-axis views will be acquired. The basal level is defined by the mitral valve, and the middle level is defined as containing some part of both papillary muscles without any mitral valve tissue through the whole cardiac cycle. Finally, the apical level is defined as the segment of the LV distal to the insertion of papillary muscles. In the process of obtaining the short-axis views, fetal heart orientation could be established using the location of liver and the $\mathrm{LV}$ position in relation to the $\mathrm{RV}^{21}$ Accordingly, there are two types of orientation for fetal heart short-axis images: (I) the liver is toward the 'left of the image' and the LV is at the 'bottom of the image', similar to the traditional transthoracic short-axis image; (II) the liver is toward the 'left of the image' and the LV is at the "top of the image" ${ }^{21}$ (figure 2). In orientation II, as viewed from the base toward the apex, the LV apex rotates in the clockwise direction and the LV base rotates counterclockwise, which are opposite directions compared with orientation I. ${ }^{21}$ Therefore, in orientation II, the rotation and twist directions of software output are inverted. In addition, in orientation II, the software output of every anterior wall should be exchanged with that of the corresponding inferior wall, respectively. Only raw data clips of cases with clear delineation of the LV and the RV wall and the septum will be included in the next step of analysis.

The raw-data clips will be stored for postprocessing offline analyses as uncompressed standard digital imaging. Standard digital imaging and communication in medicine (DICOM) files will be transferred to a workstation equipped with QLab 10.8 software (Philips Medical Systems) using automated cardiac motion quantification for offline analyses. Analyses will be performed on DICOM files to derive biventricular longitudinal strain $\left(\mathrm{S}_{\mathrm{L}}\right)$, LV circumferential strain $\left(\mathrm{S}_{\mathrm{C}}\right)$ and strain rate $\left(\mathrm{SR}_{\mathrm{L}}\right.$ and $\mathrm{SR}_{\mathrm{C}}$ ), and therefore the twist of LV. In addition, global and segmental deformation will both be investigated. Due to the inability to conduct ECG gating in fetal echocardiography, the cardiac cycle is defined by the manual selection of two consecutive end-diastolic frames (corresponding with the atrioventricular valve closure in longaxis views or $\mathrm{LV}$ chamber at its maximum size in short-axis views). No additional techniques such as dummy ECG will be used. In the left 4-chamber, long-axis and 2-chamber views, the operator will manually identify the atrioventricular lateral valve annulus and the LV apex. The software will automatically detect the endocardial borders. To ensure that the whole myocardium wall is included, the initial tracing will be manually adjusted. Next, the software will track the myocardium during the whole cardiac cycle. Tracking quality will be assessed by the observer and when necessary, the tracing will be visually verified or readjusted. When the tracking is accepted, 18 segments and 3 layers of myocardium will be created by the analysis software. Therefore, global $S_{L}$ and segmental $S_{L}$ of every layer of the myocardium will be calculated. The corresponding time to peak segmental $S_{L}$ will also be recorded. Peak segmental systolic $\mathrm{SR}_{\mathrm{L}}$, early diastolic and late diastolic $\mathrm{SR}_{\mathrm{L}}$ of every layer of the myocardium will also be obtained. The Philips software can also generate a bull's-eye polar map of global and segmental $\mathrm{S}_{\mathrm{L}}$. In the $\mathrm{LV}$ basal/mid/ apical-short-axis views, the operator will manually identify the centre and the anterior wall of LV when the chamber is at its maximum size. The software will automatically detect the endocardial borders. To ensure that the whole myocardium wall is included, and the presumptive myocardial segment is correspondent with the correct segment, the initial tracing will be manually adjusted, and the yellow arrow will be adjusted to the middle of the interventricular septum. The following steps are similar to the process of long-axis views. Eventually, the software will provide global and segmental $\mathrm{S}_{\mathrm{C}}$ of every layer of LV myocardium. The corresponding time to peak segmental $\mathrm{S}_{\mathrm{C}}$ will also be recorded. Peak segmental systolic $\mathrm{SR}_{\mathrm{C}}$, early diastolic 
and late diastolic $\mathrm{SR}_{\mathrm{C}}$ of every layer of the myocardium will also be obtained. A bull's-eye polar map of global and segmental $\mathrm{S}_{\mathrm{C}}$ can be generated. Also, the global values for left ventricular rotational displacement in degrees on apical and basal views for one complete heart cycle will be obtained. Viewing from the apex, LV counterclockwise rotation will be expressed as a positive value, whereas clockwise rotation will be expressed as a negative one. ${ }^{39} \mathrm{LV}$ twist is calculated as the net difference between apical and basal rotation (value of instantaneous apical rotation-basal rotation). In this process, the value of peak LV twist and time to peak LV twist will be obtained. Torsion is the twist value normalised to the length between the apex of the $\mathrm{LV}$ and the midpoint of the mitral valve annulus, which will be obtained from the 4-chamber view. ${ }^{21}$ Untwist, the directional reversal of LV systolic twist position during diastole is expressed as the ratio of untwist. The ratio of untwist is calculated as $[(\mathrm{a}-\mathrm{b}) / \mathrm{b} * 100 \%] /$ IVRT, where ' $\mathrm{a}$ ' is the twist at aortic valve closure and 'b' is the twist at the end of IVRT.

\section{Fetal abdominal artery ultrasound assessment}

Measurement of fetal aortic intima-media thickness (aIMT) in the upper abdomen involves obtaining a straight, non-branched $1 \mathrm{~cm}$ longitudinal segment of the proximal fetal abdominal aorta by a linear-array transducer. ${ }^{40}$ The image is focused on the posterior wall, and a resolution box function is used to magnify this part of the artery in end-diastolic cardiac phase. The angle of insonation is kept between $60^{\circ}$ to $90^{\circ}$ in a coronal or sagittal view. With this procedure, B-mode images of the proximal abdominal aorta will be obtained. Three images of the best quality will be chosen for each fetus. According to a standard protocol, ${ }^{41}$ images will be stored as standard DICOM files for offline analysis. With the assistance of a computerised programme (QLab 10.8 software, Philips Medical Systems), the offline assessment will be performed using intima-media thickness quantification. Three measurements of far-wall aIMT will be taken, and the average values of these readings will be used in the analyses. Furthermore, the aIMT results will be normalised by fetal weight.

\section{The first and second postnatal cardiovascular assessment}

The postnatal cardiovascular assessment will be acquired according to the recommendations of the American Society of Echocardiography using EPIQ 7 ultrasound systems (Philips Medical Systems) equipped with 5-12 $\mathrm{MHz}$ sector-array (12S) and 3-10 $\mathrm{MHz}$ linear-array probes $(9 \mathrm{~L})$ by the same $(\mathrm{W}-\mathrm{jB})$ operator, including an assessment of echocardiography and abdominal artery ultrasound. ${ }^{42}$ Echocardiography will be assessed with the sector-array probe, while the abdominal artery ultrasound will be acquired with the linear-array probe. The echographic images will be acquired in a quiet examination room, with the infant lying quietly awake or asleep in a supine position. Heart rates will be recorded at the time of acquisition of echography.
Echocardiography includes conventional morphometric assessment, functional assessment and STE. The process of conventional morphometric and functional assessment in postnatal period is almost the same as that in the fetus. As for STE, digital loops with five consecutive cardiac cycles triggered by the $\mathrm{R}$ wave of the QRS complex will be digitally saved, that is, apical 4-chamber, longaxis and 2-chamber views and basal/mid/apical-shortaxis views, with frame rate set at more than 80 frames/s. Raw-data clips will be stored for postprocessing offline analyses as uncompressed standard digital imaging. The following process of strain analysis in postnatal period will be almost the same as that in the fetus. Also, the process of postnatal abdominal artery ultrasound assessment is consistent with the previous statement.

\section{Statistical analysis}

Statistical Product and Service Solutions (SPSS) V.24.0 (SPSS Inc.) will be used for the statistical analysis. Data will be presented as mean $\pm \mathrm{SD}$, median (IQR) or percentages where appropriate. Independent t-test for normally distributed data and Wilcoxon rank sum test for skewed data will be used. Categorical variables will be compared using the Pearson's $\chi^{2}$ test. The association between type of conception (natural or ART) and outcomes will be estimated with linear regression by adjusting potential confounding factors. P-value $<0.05$ will be considered statistically significant.

To evaluate inter/intra-observer variability, speckle-tracking and aIMT assessments will be repeated in 15 randomly selected studies by a second observer ( $\mathrm{Y}-\mathrm{jX}$ ) blinded to the group assignment and the prior measurement and by the first observer (W-jB) after a 3-month interval, respectively. Intraclass correlation coefficients will be assessed for repeatability and reproducibility.

\section{ETHICS AND DISSEMINATION}

Little to no risk is posed to participants and their offspring in the process of this study. Written informed consent will be obtained from all participating women. Participation in the study will not interfere with the clinical intervention during pregnancy and after delivery. Findings will be disseminated through scientific publications and conference presentations.

\section{DISCUSSION}

The current high prevalence of ART for infertile couples has led to universal concerns about the impact of ART on future generations. Epidemiological studies have demonstrated an association between pathological events during fetal development and future cardiovascular risk, raising concerns about cardiovascular remodelling in fetuses conceived by ART. Well-designed prospective studies are necessary to better quantify the actual impact of ART on offspring's cardiovascular health and to identify the underlying mechanisms. 
Indeed, many studies have investigated fetal programming of cardiovascular disorders in pregnancies conceived by ART. ${ }^{415-1943-46}$ In addition, some studies focused on the differential effects of ART and situations related to ART on fetal cardiac remodelling, including small-for-gestational-age fetuses and twin pregnancies. ${ }^{47} 48$ With reference to precedent studies, the protocol of our study is mainly a replication of the study from Valenzuela-Alcaraz et $a l^{4}{ }^{4}$ The novelty of our study is adding the analysis of myocardial deformation. Few studies to date have focused on sophisticated analysis of cardiac myocardial deformation in offspring conceived by ART. The process of contraction and relaxation of the heart is extraordinarily complicated, including longitudinal contraction, circumferential contraction, radial contraction and twist-untwist process. Commonly applied evaluation methods provide only indirect information about systolic and diastolic function and no information regarding deformation of the myocardium itself. STE is a relatively new, largely angle-independent technique, based on the tracking of tissue pixels in the myocardium throughout the cardiac cycle. It has the potential to provide new insights into fetal origins of postnatal disorders. Our prospective population-based study will assess detailed cardiac mechanics in ART offspring by STE.

When this paper is accepted for publish, the enrolment and the first postnatal cardiovascular assessment will have been finished while the second follow-up will continue as planned. As a single-centre, non-randomised, prospective observational cohort study, universality of the result may be limited. It should be replicated at other centres and in other patient populations. In addition, potential loss to follow-up may lead to some loss of data. And the short follow-up period could be a limitation, but this will be overcome in our study by measuring surrogate outcomes (including ventricular longitudinal, circumferential deformation and left ventricular twist, torsion, untwist and aIMT) that are well-established precursors of future cardiovascular disorders. ${ }^{49-52}$ Finally, considering the pregnant women in the control group will be matched for maternal age with ART cases, they may be older than a normal population of healthy gravida. Whether the controls in our study can represent the normal population of healthy gravida is unknown.

Contributors $W$-jB and W- $d R$ designed the study. W-jB drafted the protocol manuscript. LC, Y-jX, GS, XW, LS and WQ significantly contributed to the refinement of study procedures and critically revised the manuscript. All authors approved the final version of the manuscript.

Funding This work was supported by the National Natural Science Foundation of China (81571686).

Competing interests None declared.

Patient consent for publication Not required.

Provenance and peer review Not commissioned; externally peer reviewed.

Open access This is an open access article distributed in accordance with the Creative Commons Attribution Non Commercial (CC BY-NC 4.0) license, which permits others to distribute, remix, adapt, build upon this work non-commercially, and license their derivative works on different terms, provided the original work is properly cited, appropriate credit is given, any changes made indicated, and the use is non-commercial. See: http:// creativecommons.org/licenses/by-nc/4.0/.

\section{ORCID iD}

Wei-dong Ren http://orcid.org/0000-0002-9555-0210

\section{REFERENCES}

1 Adamson GD, de Mouzon J, Lancaster P, et al. World collaborative report on in vitro fertilization, 2000. Fertil Steril 2006;85:1586-622.

2 Calhaz-Jorge C, De Geyter C, Kupka MS, et al. Assisted reproductive technology in Europe, 2013: results generated from European registers by ESHRE. Hum Reprod 2017;32:1957-73.

3 Heino A, Gissler M, Hindori-Mohangoo AD, et al. Variations in multiple birth rates and impact on perinatal outcomes in Europe. PLoS One 2016;11:e0149252.

4 Valenzuela-Alcaraz B, Crispi F, Bijnens B, et al. Assisted reproductive technologies are associated with cardiovascular remodeling in utero that persists postnatally. Circulation 2013;128:1442-50.

5 Basatemur E, Sutcliffe A. Follow-Up of children born after art. Placenta 2008;29 Suppl B:135-40.

6 Helmerhorst FM, Perquin DAM, Donker D, et al. Perinatal outcome of singletons and twins after assisted conception: a systematic review of controlled studies. BMJ 2004;328.

7 Schieve LA, Meikle SF, Ferre C, et al. Low and very low birth weight in infants conceived with use of assisted reproductive technology. $N$ Engl J Med 2002;346:731-7.

8 Hansen M, Bower C, Milne E, et al. Assisted reproductive technologies and the risk of birth defects--a systematic review. Hum Reprod 2005;20:328-38.

9 Basso O, Baird DD. Infertility and preterm delivery, birthweight, and caesarean section: a study within the Danish national birth cohort. Hum Reprod 2003;18:2478-84.

10 Basso O, Weinberg CR, Baird DD, et al. Subfecundity as a correlate of preeclampsia: a study within the Danish national birth cohort. Am J Epidemiol 2003;157:195-202.

11 Ertzeid G, Storeng R. The impact of ovarian stimulation on implantation and fetal development in mice. Hum Reprod 2001;16:221-5.

12 Choux C, Binquet C, Carmignac V, et al. The epigenetic control of transposable elements and imprinted genes in newborns is affected by the mode of conception: art versus spontaneous conception without underlying infertility. Hum Reprod 2018;33:331-40.

13 Barker DJ. The fetal and infant origins of disease. Eur J Clin Invest 1995;25:457-63.

14 Barker DJ, Osmond C. Infant mortality, childhood nutrition, and ischaemic heart disease in England and Wales. Lancet 1986;1:1077-81.

15 Ceelen M, van Weissenbruch MM, Vermeiden JPW, et al. Cardiometabolic differences in children born after in vitro fertilization: follow-up study. J Clin Endocrinol Metab 2008;93:1682-8.

16 Meister TA, Rimoldi SF, Soria R, et al. Association of assisted reproductive technologies with arterial hypertension during adolescence. J Am Coll Cardiol 2018;72:1267-74.

17 Scherrer U, Rimoldi SF, Rexhaj E, et al. Systemic and pulmonary vascular dysfunction in children conceived by assisted reproductive technologies. Circulation 2012;125:1890-6.

18 Valenzuela-Alcaraz B, Serafini A, Sepulveda-Martínez A, et al. Postnatal persistence of fetal cardiovascular remodelling associated with assisted reproductive technologies: a cohort study. BJOG 2019;126:291-8.

19 Rexhaj E, Paoloni-Giacobino A, Rimoldi SF, et al. Mice generated by in vitro fertilization exhibit vascular dysfunction and shortened life span. J Clin Invest 2013;123:5052-60.

20 Miranda JO, Cerqueira RJ, Ramalho C, et al. Fetal Cardiac Function in Maternal Diabetes: A Conventional and Speckle-Tracking Echocardiographic Study. J Am Soc Echocardiogr 2018;31:333-41.

21 Li L, Craft M, Hsu HH, et al. Left Ventricular Rotational and Twist Mechanics in the Human Fetal Heart. J Am Soc Echocardiogr 2017;30:773-80.

$22 \mathrm{He}$ J-R, Li W-D, Lu M-S, et al. Birth weight changes in a major City under rapid socioeconomic transition in China. Sci Rep 2017;7:1031.

23 Dupont WD, Plummer WD. Power and sample size calculations. A review and computer program. Control Clin Trials 1990;11:116-28.

24 Dupont WD, Plummer WD. Normal values of Pulsatility index from fetal vessels: a crosssectional study on 1556 healthy fetuses. Control Clin Trials 1990;11:116-28.

25 del Río M, Martínez JM, Figueras F, et al. Reference ranges for Doppler parameters of the fetal aortic isthmus during the second half of pregnancy. Ultrasound Obstet Gynecol 2006;28:71-6. 
26 Gómez O, Figueras F, Fernández S, et al. Reference ranges for uterine artery mean Pulsatility index at 11-41 weeks of gestation. Ultrasound Obstet Gynecol 2008;32:128-32.

27 Hecher K, Campbell S, Snijders R, et al. Reference ranges for fetal venous and atrioventricular blood flow parameters. Ultrasound Obstet Gynecol 1994;4:381-90.

28 Baschat AA, Gembruch U. The cerebroplacental Doppler ratio revisited. Ultrasound Obstet Gynecol 2003;21:124-7.

29 Teichholz LE, Kreulen T, Herman MV, et al. Problems in echocardiographic volume determinations: Echocardiographicangiographic correlations in the presence or absence of asynergy. Am J Cardiol 1976;37:7-11.

30 Lowes BD, Gill EA, Abraham WT, et al. Effects of carvedilol on left ventricular mass, chamber geometry, and mitral regurgitation in chronic heart failure. Am J Cardiol 1999;83:1201-5.

31 Schneider C, McCrindle BW, Carvalho JS, et al. Development of $Z$-scores for fetal cardiac dimensions from echocardiography. Ultrasound Obstet Gynecol 2005;26:599-605.

32 DeVore GR, Klas B, Satou G, et al. 24-segment sphericity index: a new technique to evaluate fetal cardiac diastolic shape. Ultrasound Obstet Gynecol 2018;51:650-8.

33 Lang RM, Badano LP, Mor-Avi V, et al. Recommendations for cardiac chamber quantification by echocardiography in adults: an update from the American Society of echocardiography and the European association of cardiovascular imaging. Eur Heart $J$ Cardiovasc Imaging 2015;16:233-71.

34 Kiserud T, Ebbing C, Kessler J, et al. Fetal cardiac output, distribution to the placenta and impact of placental compromise. Ultrasound Obstet Gynecol 2006;28:126-36.

35 Gardiner HM, Pasquini L, Wolfenden J, et al. Myocardial tissue Doppler and long axis function in the fetal heart. Int $J$ Cardiol 2006;113:39-47.

36 Comas M, Crispi F, Gómez O, et al. Gestational age- and estimated fetal weight-adjusted reference ranges for myocardial tissue Doppler indices at 24-41 weeks' gestation. Ultrasound Obstet Gynecol $2011 ; 37: 57-64$.

37 DeVore GR. Assessing fetal cardiac ventricular function. Seminars in Fetal and Neonatal Medicine 2005;10:515-41.

38 Meriki N, Izurieta A, Welsh A. Reproducibility of constituent time intervals of right and left fetal modified myocardial performance indices on pulsed Doppler echocardiography: a short report. Ultrasound Obstet Gynecol 2012;39:654-8.

39 Omar AMS, Vallabhajosyula S, Sengupta PP. Left ventricular twist and torsion: research observations and clinical applications. Circ Cardiovasc Imaging 2015;8:e003029.
40 McCloskey K, Vuillermin P, Carlin JB, et al. Perinatal microbial exposure may influence aortic intima-media thickness in early infancy. Int J Epidemiol 2017;46:209-18.

41 Di Bernardo S, Mivelaz Y, Epure AM, et al. Assessing the consequences of gestational diabetes mellitus on offspring's cardiovascular health: MySweetHeart cohort study protocol, Switzerland. BMJ Open 2017;7:e016972.

42 Lopez L, Colan SD, Frommelt PC, et al. Recommendations for quantification methods during the performance of a pediatric echocardiogram: a report from the pediatric measurements writing group of the American Society of echocardiography pediatric and congenital heart disease Council. Journal of the American Society of Echocardiography 2010;23:465-95.

43 Zhou J, Liu H, Gu H-tao, et al. Association of cardiac development with assisted reproductive technology in childhood: a prospective single-blind pilot study. Cell Physiol Biochem 2014;34:988-1000.

44 Liu H, Zhang Y, HT G, et al. Association between art and cardiac alteration at age 5 years. JAMA Pediatr 2015;169:603-5.

45 von Arx R, Allemann Y, Sartori C, et al. Right ventricular dysfunction in children and adolescents conceived by assisted reproductive technologies. J Appl Physiol 2015;118:1200-6.

46 Guo X-Y, Liu X-M, Jin L, et al. Cardiovascular and metabolic profiles of offspring conceived by assisted reproductive technologies: a systematic review and meta-analysis. Fertil Steril 2017;107:622-31.

47 Valenzuela-Alcaraz B, Crispi F, Cruz-Lemini M, et al. Differential effect of assisted reproductive technology and small-for-gestational age on fetal cardiac remodeling. Ultrasound Obstet Gynecol 2017;50:63-70.

48 Valenzuela-Alcaraz B, Cruz-Lemini M, Rodríguez-López M, et al. Fetal cardiac remodeling in twin pregnancy conceived by assisted reproductive technology. Ultrasound Obstet Gynecol 2018;51:94-100.

49 Ternacle J, Bodez D, Guellich A, et al. Causes and consequences of longitudinal LV dysfunction assessed by 2D strain echocardiography in cardiac amyloidosis. JACC: Cardiovascular Imaging 2016;9:126-38.

50 Barros-Gomes S, Eleid MF, Dahl JS, et al. Predicting outcomes after percutaneous mitral balloon valvotomy: the impact of left ventricular strain imaging. Eur Heart J Cardiovasc Imaging 2017;18:763-71.

51 Motoki H, Koyama J, Nakazawa H, et al. Torsion analysis in the early detection of anthracycline-mediated cardiomyopathy. Eur Heart $J$ Cardiovasc Imaging 2012;13:95-103.

52 Dawson JD, Sonka M, Blecha MB, et al. Risk factors associated with aortic and carotid intima-media thickness in adolescents and young adults. J Am Coll Cardiol 2009;53:2273-9. 